\title{
Fortgeschrittenes Mammakarzinom
}

\section{Everolimus kann den klinischen Nutzen der antihormonellen Therapie verstärken}

Dies schlussfolgerte Prof. Dr. José Baselga, Boston/USA, aus den Ergebnissen der Studie BOLERO-2 (Breast Cancer Trials of Oral Everolimus-2). Auf Basis der in der BOLERO-2-Studie gezeigten Wirksamkeit zählt Everolimus zu den wichtigsten Fortschritten im Bereich der medizinischen Onkologie, so Prof. Dr. Fabrice André, Villejuif/Frankreich, in der Diskussion der Studienergebnisse.

In die Phase-III-Studie waren 724 postmenopausale Patientinnen mit fortgeschrittenem, ER (Östrogenrezeptor)-positivem, HER2 (humaner epidermaler Wachstumsfaktorrezeptor 2)-negativem Mammakarzinom eingeschlossen. Beiallen Patientinnen war die Tumorerkrankung nach oder während einer antihormonellen Therapie mit Anastrozol oder Letrozol wieder aufgetreten oder fortgeschritten. Randomisiert erhielten 485 Patientinnen Everolimus (10mg/Tag) plus Exemestan (25mg/Tag) und 239 Patientinnen Placebo plus Exemestan (25mg/ Tag) bis zur Progression oder dem Auftreten nicht tolerierbarer Toxizität. Ein Cross-over war nicht möglich. Die Charakteristika der Patientinnen waren in der Ausgangssituation laut Baselga gut ausgeglichen.

\section{Progressionsfreies Überleben „klinisch bedeutsam“ verlängert} Bereits in der vorab geplanten Interimsanalyse erreichte die Studie den primären Endpunkt. In der primären Wirksamkeitsanalyse durch die Prüfärzte ergab sich eine laut Baselga „klinisch bedeutsame“ Verlängerung des medianen progressionsfreien Überlebens (Abb.). Die zentrale Beurteilung der Daten bestätigte dies. Der Vorteil zugunsten derTherapie mit Everolimus/Exemestan wurde in allen vorab definierten Subgruppen beobachtet.

Eine im Vergleich zur Behandlung mit Placebo/Exemestan beobachtete Zunahme unerwünschter Wirkungen unter Everolimus/Exemestan führte aufgrund des anhaltenden klinischen Nutzens nicht zu einer Beeinträchtigung der Lebensqualität bei den in der Studie verbliebenen Patientinnen, so Baselga. Es wurden keine bislang nicht bekannten unerwünschten Wirkungen der Therapie mit Everolimus berichtet. Häufigste Grad-3/4-Nebenwirkungen waren laut Baselga Stomatitis, Hyperglykämie, Fatigue und Pneumonitis.

\section{Rationale für die mTOR-Inhibition - Ausblick}

Die Rationale für die Kombination des mTOR (mammalian Target of Rapamycin)-Inhibitors Everolimus mit dem Aromataseinhibitor Exemestan sind Wechselwirkungen zwischen dem ER- und dem mTORSignalweg. Bekannt ist laut Baselga eine ligandenunabhängige ERAktivierung durch den mTOR-Signalweg. Östradiol wiederum kann über eine Blockade des PI3K (Phosphoinositol-3-Kinase)/mTOR-Signalwegs Apoptose unterdrücken. Darüber hinaus wurde in präklinischen Untersuchungen bei Resistenz gegen eine antihormonelle Therapie eine Hyperaktivierung des PI3K/mTOR-Signalwegs beobachtet.

Ausgehend von den bislang bekannten Wechselwirkungen des ER- und mTOR-Signalwegs und der möglichen Aktivierung paralleler bzw. kompensatorischer Signalwege zeigte André Perspektiven für eine weitere Verbesserung der Behandlung bei ER-positivem Mammakarzinom auf. Erste positive Daten liegen so z.B. zur Kombination mit IGFR1 (Insulin-ähnlicher Wachstumsfaktorrezeptor 1)-Inhibitoren vor. Für die Zukunft würde dies jedoch auch bedeuten, so André, dass sequenzielle Biopsien im Behandlungsverlauf erforderlich sind, um

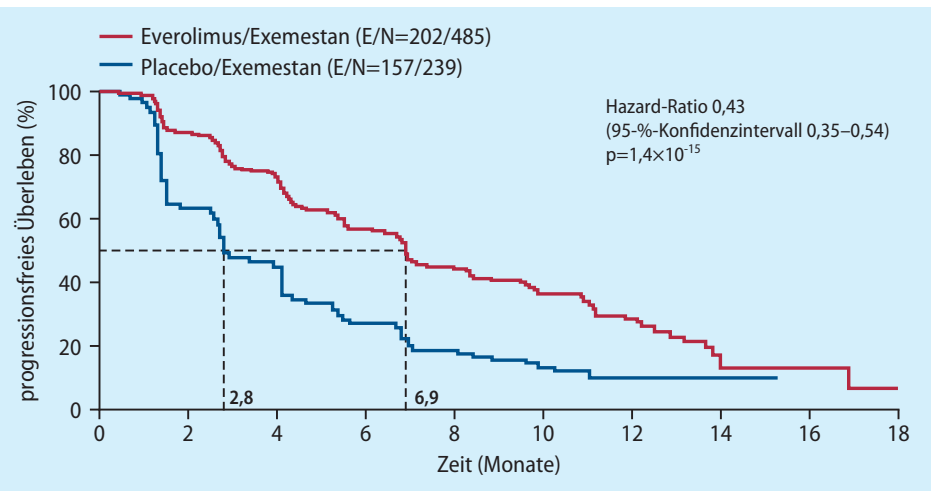

nach Baselga J et al., 2011, EMCC, LBA9, oral presentation

BOLERO-2-Studie - Everolimus/Exemestan versus Placebo/Exemestan: Progressionsfreies Überleben, Auswertung durch die Prüfärzte

die Therapie auf Basis des molekularen Profils zu individualisieren und damit das Therapieergebnis weiter zu verbessern. $A M$

Presidential Session III, Baselga J et al., 2011, EMCC: 9LBA, oral presentation

\section{Metastasiertes Kolorektalkarzinom Neue Therapieoption mit Aflibercept}

In vorab geplanten Subanalysen konnte die in einer Phase-III-Studie gezeigte Wirksamkeit von Aflibercept bei metastasiertem Kolorektalkarzinom bestätigt werden: Es profitierten alle Patienten.

In der VELOUR-Studie erreichten mit Oxaliplatin vorbehandelte Patienten unter Aflibercept plus FOLFIRI (Folinsäure/5-Fluorouracil/lrinotecan) ein signifikant längeres Gesamtüberleben und ein signifikant längeres progressionsfreies Überleben als unter FOLFIRI allein ( $p=0,0032$ bzw. $p=0,00007$; van Cutsem E et al., 2011, WCGC: O-0024, oral presentation). In vorab geplanten Subanalysen wurden Stratifizierungsfaktoren (Allgemeinzustand, vorangegangene Therapie mit Bevacizumab) und Patientencharakteristika (Alter, Geschlecht, Herkunft, Hypertonie, Metastasenanzahl, auf die Leber begrenzte Erkrankung, Lokalisation des Primärtumors) berücksichtigt. Dabei konnte der positive Effekt von Aflibercept auf das Gesamtüberleben und das progressionsfreie Überleben in allen Subgruppen gezeigt werden.

Aflibercept ist ein Fusionsprotein aus extrazellulären VEGF (Vascular Endothelial Growth Factor)-Rezeptordomänen und dem FcTeil des humanen Immunglobulins $G$. Es bindet an Wachstumsfaktoren, wie z.B. VEGF-A und -B sowie PIGF (Placental Growth Factor), die u.a. für die Tumorangiogenese eine Rolle zu spielen scheinen, mit höherer Affinität als deren natürliche Rezeptoren. AM

Presidential Session II, Tabernero J et al., 2011, EMCC: 6LBA, oral presentation 